\title{
Gemeinsam und gezielt etwas ändern
}

\section{Daniel Lüthi}

Freier Journalist und Fotograf, Medientrainer, Bern

\begin{abstract}
Alle hätten das gleiche Recht auf Gesundheit. Aber die Realität ist anders: Wer sozial benachteiligt ist, ist es auch gesundheitlich. Das 20. forumsante.ch in Bern hat diese Tatsache mehrfach bestätigt - aber auch einige Lösungsansätze aufgezeigt.
\end{abstract}

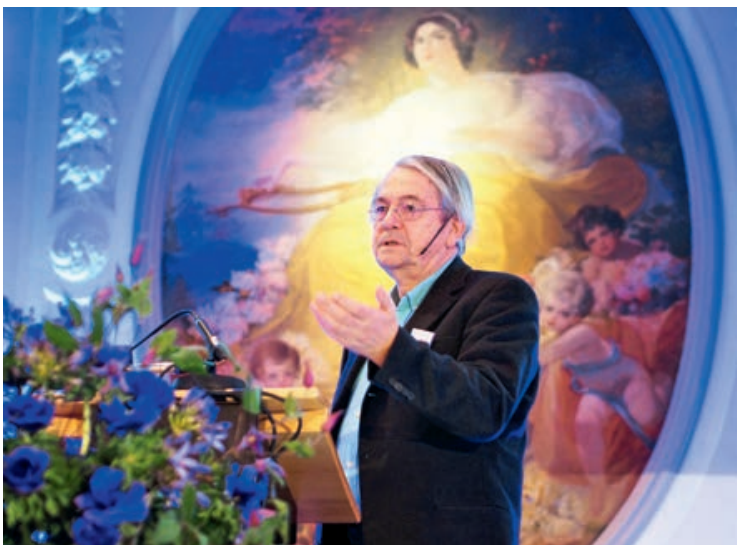

Prof. Thierry Lang (Toulouse).

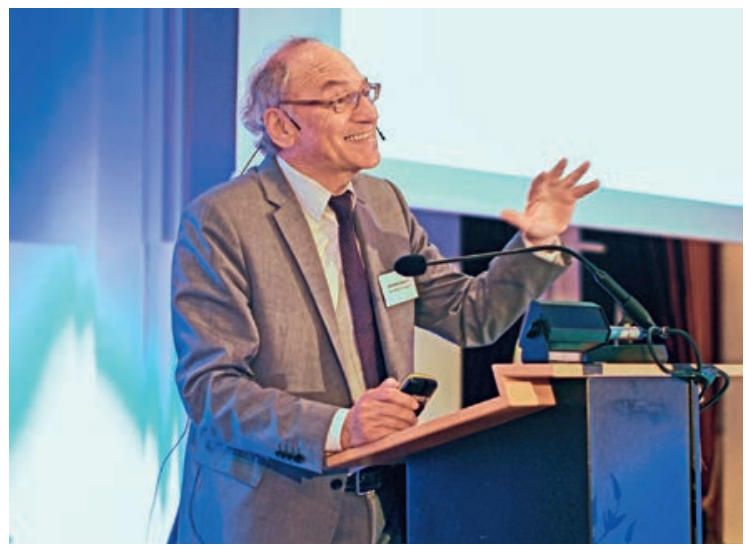

Prof. Johannes Siegrist (Düsseldorf).

\section{Résumé}

Tout le monde aurait le même droit à la santé. Mais la réalité est différente: les personnes socialement défavorisées le sont également au niveau de la santé. Le $20^{\mathrm{e}}$ forumsante.ch à Berne l'a confirmé à plusieurs reprises, mais a également mis un certain nombre de solutions en évidence.
«Sozioökonomische Determinanten» ist ein nebulöser Fachbegriff, hinter dem sich eine klare Ungerechtigkeit verbirgt. «Sag mir, wer du bist, und ich sage dir, wie lange du lebst»: So fasste es die Einladung zu dieser Tagung zusammen. Fünf Sterne und eine startbereite Luxuskarosse empfingen vor dem Nobelhotel die interessierten Gesundheitsfachleute - «währenddem wir hier sicher sitzen, haben Bauarbeiter draussen ein grösseres Risiko, heute zu verunfallen", stellte drinnen einer der Referenten fest, nämlich Marc Höglinger, Versorgungsforscher an der ZHAW.

\section{Soziale Ungleichgewichte beeinflussten die Mortalität}

Zwischen Handwerkern und Intellektuellen gebe es markante Unterschiede bei der Mortalität, bestätigte Thierry Lang, Professor an der Universität Toulouse. Biomedizinische Risikofaktoren wie hoher Blutdruck, Übergewicht, Tabak- oder Alkoholkonsum seien das eine, sagte er. Als unerträglich erachte er die sozialen Ungleichgewichte, die diese Faktoren beeinflussen: verschiedene Einkommen, unterschiedliche Bildung, Bindungen und Beziehungen.

"Je höher du stehst in der Gesellschaft, umso besser ist deine Gesundheit»: Dies sei geradezu eine Binsenwahrheit, sagte Johannes Siegrist, Schweizer Professor am Institut für Medizinische Soziologie an der Universitätsklinik Düsseldorf. Er zitierte Studien aus der modernen Arbeitswelt, die zwei spezielle Faktoren betonen: Autonomie und Anerkennung. Leistungsdruck und wirtschaftliche Zwänge führen zu Ausbeutung und schränken die Autonomie ein, war eine seiner Hauptaussagen. Die andere: Gewinnstreben geht auch auf Kosten von Anerkennung. «Menschen geben ihr Bestes, aber es kommt kaum etwas zurück.» Das habe Stress zur Folge und mache physisch und psychisch 


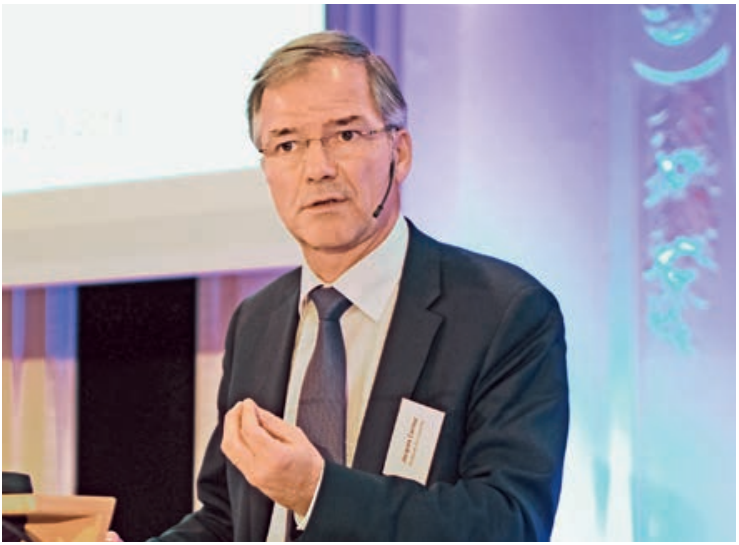

Prof. Jacques Cornuz (Lausanne).

krank. Koronare Herzkrankheiten und Depressionen stehen im Vordergrund

\section{Was tun?}

Die Fakten also sind längst bekannt und wissenschaftlich bestens dokumentiert. Immer mehr kristallisierte sich denn auch an dieser Tagung die Frage heraus, wie und wo wir diese unerträglichen Ungleichgewichte und Ungerechtigkeiten wirkungsvoll bekämpfen können. Pfannenfertige Lösungen könne und wolle das forumsante.ch nicht anbieten, aber Anregungen zum Weiterdenken, sagte Mitorganisator Jacques de Haller schon in seinem Eröffnungsvotum. Es war beispielsweise Jacques Cornuz vom Zentrum für Allgemeinmedizin und Public Health der Universität Lausanne, der einen konkreten Ansatz präsentierte. Er stellte das Projekt «Alliance santé» vor, einen Zusammenschluss von vier Institutionen im Kanton Waadt zu einem ein- zigen Kompetenzzentrum mit rund 800 Mitarbeitenden, darunter 200 Ärzte/-innen und 300 Pflegende.

\section{«Die Politik muss die Entwicklung des Kindes fördern", sagt Thierry Lang.}

Grundidee: der interprofessionelle, partnerschaftliche Ansatz. Medizin und Politik, die gemeinsam aktiv sind. Und gezielt, wie Stéfanie Monod im Gespräch mit der SÄZ ergänzte. Monod ist Ärztin und im Kanton Waadt für die öffentliche Gesundheit verantwortlich. Sie sass in Bern im Publikum und konkretisierte den erwähnten Lösungsansatz so: «Prävention beispielsweise betreiben wir nicht mehr nach dem Giesskannenprinzip, sondern fokussiert, so in speziellen Quartieren, wo besonders viele benachteiligte Menschen leben.»

Und weil in den einzelnen Referaten für Lösungsansätze oft keine Zeit mehr blieb, sprachen wir auch Thierry Lang aus Toulouse speziell darauf an. Gefragt seien Interventionen an der Wurzel, in der Kindheit, der Familie, der Schule, sagte er. «Die Politik muss die Entwicklung des Kindes fördern, beispielsweise mit genügend und guten Angeboten, um Kinder extern zu betreuen.» Lang war aber auch für eine direkte Verbindung mit der Aktualität besorgt. Gegenüber der SÄZ sagte er, es gehe nicht bloss darum, die "grosse Armut» zu bekämpfen. Der Unmut der Gelbwesten in seinem Heimatland Frankreich sei das klare Signal einer benachteiligten Mittelschicht, dass sie nicht länger bereit sei, die im Fünfsternehaus diskutierten Ungleichheiten zu akzeptieren.

\section{Bildnachweise}

Fotos der Vortragenden: Daniel Lüthi

Foto Hotel Bellevue Bern: @ Denis Linine | Dreamstime.com (bearbeitet von die Medienmacher AG)

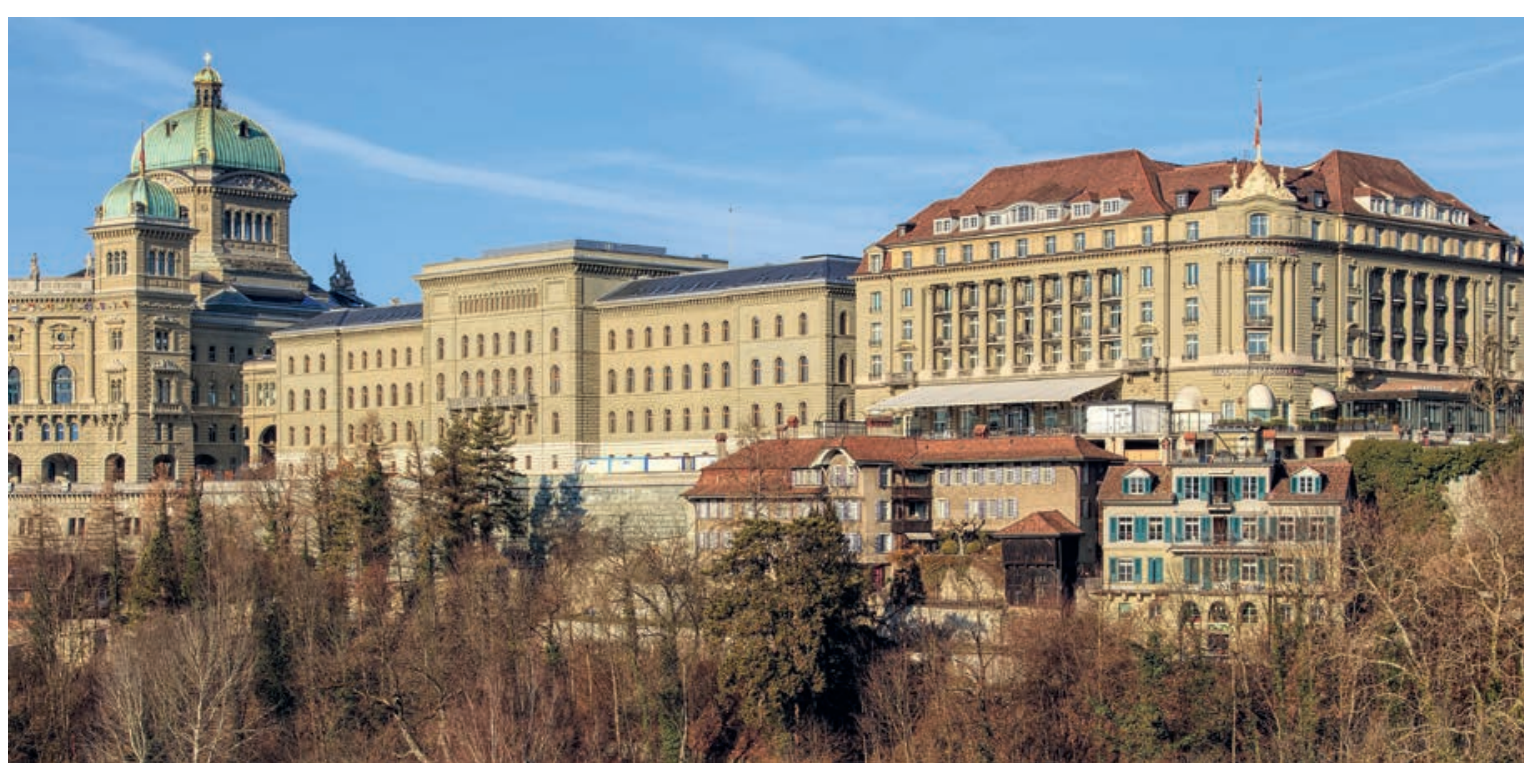

Das 20. forumsante.ch fand am 15. Januar 2019 im Hotel Bellevue in Bern statt. 